\title{
CARACTERÍSTICAS GEOTÉCNICAS E HIDROGEOLÓGICAS DE SITIOS PROPUESTOS COMO RELLENOS SANITARIOS EN COSTA RICA
}

\author{
Rolando Mora \& Asdrúbal Vargas \\ Escuela Centroamericana de Geología \\ Apdo. 214-2060, Universidad de Costa Ricqa \\ E-Mail: rolandom@geologia.ucr.ac.cr/AVargasS@icelec.ice.go.cr
}

(Recibido 3/10/2000; Aceptado 27/3/2001)

\begin{abstract}
In this paper the geological, soil mechanical, and hydrogeological conditions of six propoused landfills were investigated and also the polution potencial of the groundwater was estimated with the Le Grand methodology. The analysed sites are Paraiso, Oreamuno, Santo Domingo, Atenas, Puriscal, Bolivar y Cerro Botadero; the majority are placed in the Central Valley of Costa Rica, involving volcanic materials.

The landfill Santo Domingo is receiving solid wastes since 10 years ago, this place is very close to the La Valencia well fields and La Libertad springs, which are used to supply potable water to the city of San Jose; therefore the need for protection of the groundwater against polution is very important. As a first recommendation, various piezometers must be constructed near to the site, for the monitoring the groundwater quality.

The residual soils of the Peña Negra Formation can be used for the impermeabilization of the Pozos landfill in Puriscal; the hydraulic conductivity of the materials of the upper part of the soil-profil vary from $5 \times 10-4$ to 5 $\mathrm{x} 10-6 \mathrm{~cm} / \mathrm{s}$, but the underlying materials are very fractured and their hydraulic conductivity can be higher; we recomend an adecuate soil seal at the base.

In Atenas site, the wastes and leachate must be handled with care to prevent the contamination of the streams. The Cerro Botadero site have a high polution potential, because the groundwater table is very close to the surface, and the materials of the aquifer are gravel and sand, with moderate hydraulic conductivity. The monitoring of the water quality in the La Gata and Seca streams is recomended and an adecuate impermeabilization at the base.
\end{abstract}

RESUMEN: En este trabajo se analizan seis localidades donde se pretende construir un relleno sanitario. El sitio Santo Domingo, reviste gran importancia, ya que se ubica cerca del campo de pozos de la Valencia y de los manantiales de la Libertad y se encuentra en funcionamiento desde hace varios años. Como una primera medida de protección del agua subterránea se deberían construir piezómetros de monitoreo, gradiente abajo del sitio, para evaluar periódicamente la calidad del agua. Los suelos residuales de la Formación Peña Negra, en el sitio Pozos de Puriscal, pueden utilizarse para impermeabilizar, su resistencia al corte en estado compactado es media y su resistencia a la compresibilidad es alta y poco permeables. En el caso del sitio Atenas deben manejarse con sumo cuidado la disposición y manejo de los desechos, para evitar la fuga de lixiviados y la contaminación directa de las quebradas que nacen en la fila Boquerón. El cerro Botadero presenta el potencial de contaminación más alto, ya que a cierta distancia se encuentra un acuífero superior, el nivel de agua se encuentra relativamente cerca de la superficie y la ubicación del sitio es desfavorable, se recomienda realizar una impermeabilización y manejo de desechos adecuado. 


\section{INTRODUCCIÓN}

La ubicación de un relleno sanitario requiere que se consideren varias condiciones, entre ellas las económicas, las sociales y las geoambientales; estas últimas, a su vez, incluyen características geológicas, hidrogeológicas y geotécnicas.

En los últimos años varias municipalidades y entidades privadas de nuestro país han considerado la posibilidad de construir rellenos sanitarios, como medida para atender la creciente necesidad de disponer los desechos sólidos. Por ejemplo, la municipalidad de Garabito construyó un relleno sanitario con un costo superior a los $\notin 40000000$ y la municipalidad de Corredores construyó un relleno en el cual invirtió $\varnothing 20000000$ (La Nación, 15 agosto 2000), así mismo en las localidades de San Carlos, Turrialba y San Ramón, entre otros, se están implementando medidas similares (La Nación, 12 de agosto de 2000).

En este trabajo se recopila información sobre parámetros geológicos, geotécnicos e hidrogeológicos de varios sitios propuestos como rellenos sanitarios. A partir de estos resultados se estima que varios de ellos ofrecen condiciones naturales adecuadas de impermeabilización y de resistencia al corte de los taludes, sin embargo, para otros se deben realizar trabajos de compactación y de impermeabilización adecuados.

El objetivo de este trabajo es recopilar información sobre la geología y las características geomécanicas e hidrogeológicas de siete sitios propuestos como rellenos sanitarios. Además se pretende analizar el potencial de contaminación del agua subterránea, por medio de la metodología de Le Grand (Todd, 1976). Los sitios analizados se muestran en la figura 1, siendo denominados Paraíso (RSP), Oreamuno (OR), Santo Domingo (RSD), Pozos (RSPU), Atenas(RATE), Bolívar (RSR) y cerro Botadero (RSCB), la mayoría de ellos se ubican en el Valle Central (Cuadro 1).

La metodología de trabajo ha incluido trabajo de campo, en el cual se han realizado ensayos de permeabilidad, ensayos de penetra-

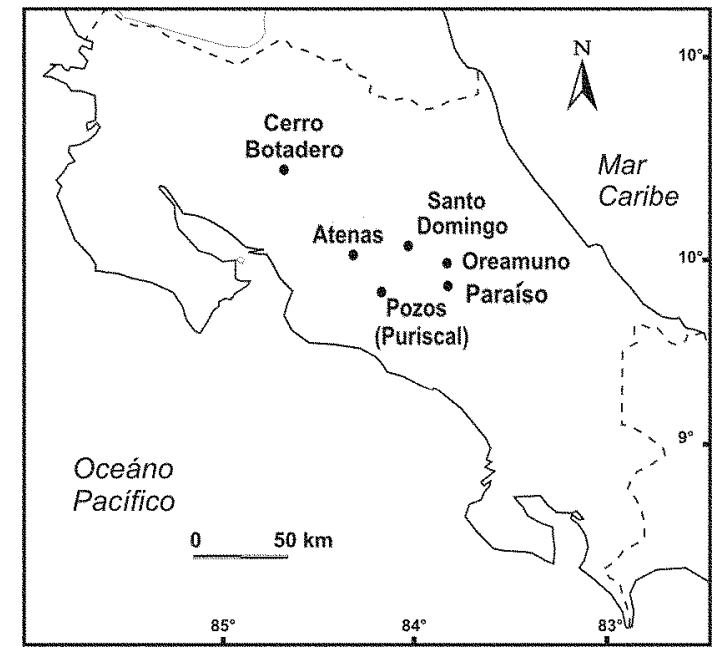

Fig. 1: Ubicación de los sitios investigados en Costa Rica.

Cuadro 1

Ubicación de los sitios investigados

\begin{tabular}{lcc}
\hline Sitio propuesto & Latitud & Longitud \\
\hline Oreamuno & $206,20 \mathrm{~N}$ & $549,70 \mathrm{E}$ \\
Paraíso & $203,75 \mathrm{~N}$ & $555,00 \mathrm{E}$ \\
Sto. Domingo & $261,80 \mathrm{~N}$ & $526,40 \mathrm{E}$ \\
Pozos, Puriscal & $196,50 \mathrm{~N}$ & $504,75 \mathrm{E}$ \\
Atenas & $216,25 \mathrm{~N}$ & $495,100 \mathrm{E}$ \\
Bolivar, San Ramón & $232,00 \mathrm{~N}$ & $483,00 \mathrm{E}$ \\
Cerro Botadero, Las Juntas & $250,50 \mathrm{~N}$ & $433,80 \mathrm{E}$
\end{tabular}

ción, reconocimiento geológico y toma de muestras de suelo. Además, se han realizado análisis de laboratorio, recopilación de información bibliográfica y trabajo de gabinete, lo que ha permitido integrar toda la información disponible.

Es realmente escaso el número de publicaciones sobre las condiciones geológicas, geomécanicas e hidrogeológicas de sitios potenciales para instalar rellenos sanitarios en Costa Rica. Knoblich \& Mands (1993) analizan las características del sitio cabezas en Esparza y del cerro Asilo conocido también como Río Azul. Mora (1998) evalua las propiedades físico-mecánicas e hidráulicas de suelos de origen volcánico en varios sitios seleccionados. 


\section{CARACTERÍSTICAS GEOLÓGICAS DE LOS SITIOS PROPUESTOS}

El sitio denominado Oreamuno, que se ubica en el cantón de Oreamuno, en la provincia de Cartago, se encuentra geológicamente en el Miembro Superior de la Formación Reventado, el cual está compuesto por coladas de lava andesítica, lahares y capas de ceniza y tiene un espesor de $600 \mathrm{~m}$. Localmente, se observa una capa de cenizas de 4,4 m de espesor, la que es subyacida por un lahar de 3,2 m, el cual es subyacido por un colada de lava meteorizada, con un espesor observable de 3,4 m.

El sitio Paraiso se localiza en el cantón de Paraiso y se encuentra caracterizado por el Miembro Cama de Ceniza de la Formación Reventado, el cual está compuesto por cenizas de color rojo, de grano fino y muy meteorizadas (Krushensky, 1972). Las cenizas finas, muy meteorizadas se encuentran hasta profundidades de 3,2 m, también aparecen coladas de lava meteorizadas, a profundidades variables y bloques de lavas andesíticas en las partes superiores del perfil de suelos.

En el sitio Santo Domingo se observan tobas de la Formación Tiribí con un espesor aproximado de $30 \mathrm{~m}$. Subyaciendo estos materiales se encuentran lavas densas y brechosas del Miembro La Libertad, descritas por (Echandi, 1981) como Miembro Linda Vista de la Formación Colima, con un espesor aproximado de $90 \mathrm{~m}$ y que dan origen al acuífero La Libertad. Posteriormente se encuentra un paquete de tobas arcillosas de $15 \mathrm{~m}$ y subyaciendo esta unidad se presenta el Miembro Colima Superior de la Formación Colima (Ibid, 1981).

El sitio Pozos pertenece al cantón de $\mathrm{Pu}$ riscal y geológicamente se identifica dentro de la Formación Peña Negra, descrita por Denyer \& Arias (1991). Arias (1988) menciona que el espesor, de la formación es de aproximadamente $1000 \mathrm{~m}$ y la divide en tres secciones: una secuencia de conglomerados brechosos hacia el techo, interestratificada con una arenisca fosilífera parda; la segunda es una secuencia de lutitas negras y la tercera compuesta de areniscas brechosas y lavas. Las tres unidades mencionadas han sido identificadas en el sitio. En las calicatas excavadas para la exploración de los suelos se ha identificado un espesor de suelo de tipo residual de 0 a 2,3 m. En los alrededores del sitio han sido identificados cuatro lineamientos sugestivos de ser fallas recientes (Montero, 1994). Esta región presentó una secuencia de temblores que se inició en mayo de 1990 y que incluyó varios temblores de magnitudes entre 4,5 y 4,8, asociándose la mayoría de ellos a la falla San Antonio y Picagres (Idem). Por su parte la aceleración máxima del suelo en los alrededores del sitio puede alcanzar $29 \%$ de la aceleración de la gravedad (Morales \& Aguilar, 1994).

El sitio Atenas pertenece al cantón de Atenas. Los materiales encontrados en el sitio comprenden $0,2 \mathrm{~m}$ de materia orgánica, subyacida por un suelo residual con espesor de 1,5-2,4 $\mathrm{m}$ y luego aparecen lavas andesíticas de la Formación Grifo Alto (Denyer \& Arias, 1990) en un estado de meteorización muy avanzado, del cual se aprecia un espesor de 3,4 m).

El sitio Bolivar pertenece al cantón de San Ramón y se correlaciona geológicamente con la unidad Piroclástica de Caída (Alvarado, 1994) y está constituida por tobas de caída poco consolidadas y muy alteradas. Este autor considera un espesor mínimo de $50 \mathrm{~m}$ para esta unidad.

El terreno denominado cerro Botadero se encuentra al sureste de las Juntas de Abangares. En el sitio se excavaron tres calicatas para explorar los suelos y la geología. En la calicata 1 se observa un suelo orgánico de color negro, el cual es subyacido por un coluvio de un metro de espesor, compuesto por bloques de rocas volcánicas e inmersos en una matriz arcillosa. Luego aparece una arenisca fina. En la calicata 2 aparece, el coluvio con un espesor de $1,35 \mathrm{~m}$, el cual es subyacido por areniscas finas de color crema. Esta areniscas fueron descritas por Madrigal (1980) como Lacustre Portones. En la calicata 3 se observó de nuevo el suelo orgánico de $0,3 \mathrm{~m}$ de espesor subyacido por un limo, de color rojo de 1,5 $\mathrm{m}$ de espesor y una arenisca fina. 


\section{CARACTERÍSTICAS GEOTÉCNICAS DE LOS SITIOS PROPUESTOS}

Para los casos que nos ocupan los valores de límite líquido oscilan de 39,1-70,1\%, los valores de límite plástico de 22,4-49,0\% y el índice de plasticidad desde 16,7-24,4\% (Cuadro 2). En los casos donde los materiales no son adecuados para las construcción del sistema de impermeabilización, estos se pueden mejorar mediante mezclas de suelos apropiados. La relación de vacíos oscila entre 0,41 en Pozos hasta 2,4 en el sitio Oreamuno. Por su parte los contenidos de humedad varían de 9,1\% en el sitio Pozos hasta 57,5\% en el cerro Botadero.

Knoblich \& Mands (1993) proponen que la impermeabilización geológica de la base de un relleno sanitario debe tener una conductividad hidráulica compactada menor de $1 \times 10^{-5} \mathrm{~m} / \mathrm{s}$ o < $1 \times 10^{-7} \mathrm{~m} / \mathrm{s}$ y un límite líquido de $35-45 \%$, el límite plástico de $20-45 \%$ y el índice de plasticidad de $10-25 \%$ los cuales son característicos de arcillas de baja plasticidad o suelos mezclados con 30-40\% de arcilla/limo.

Los valores de permeabilidad de los sitios investigados se encuentran en el orden de $1 \times 10^{-5}$ $-5 \times 10^{-8} \mathrm{~m} / \mathrm{s}$, y las permeabilidades compactadas alcanzan en la mayoría de los casos valores de $10^{-10} \mathrm{~m} / \mathrm{s}$; sin embargo, una muestra del cerro Botadero, no alcanzó el valor requerido.

\section{CARACTERÍSTICAS HIDROGEOLÓGICAS}

Para el sitio Oreamuno se han identificado 6 pozos que cuentan con información: IS-226, Is304, Is-308, Is-309, Is-404 e Is-429, los cuales suplen de agua subterránea actividades agroindustriales y domésticas (Cuadro 3). El nivel estático reportado oscila entre 7 y $147 \mathrm{~m}$ de profundidad, la mayoría de mediciones son niveles potenciométricos. El caudal de extracción varía de 1,2 a 14 1/s, coincidiendo este último con el pozo de mayor profundidad, el cual posiblemente capta un nivel regional, mientras que los demás pozos captan acuíferos colgados. La transmisividad varía de 10 a $1244 \mathrm{~m}^{2} / \mathrm{d}$, con un promedio de $20 \mathrm{~m}^{2} / \mathrm{d}$ y con una capacidad específica de 0,15 a 9,4 1/s/m. Las perforaciones tienen profundidades que oscilan de 34 a 200 m, siendo más frecuentes las perforaciones con profundidades menores de $100 \mathrm{~m}$.

A partir de la información que brindan las descripciones de las perforaciones, se puede asumir que en el área se presentan lahares constituidos por fragmentos de lava de tamaño variable, envueltos en una matriz arcillosa y en menor proporción de arena. La mayoría de los pozos inventariados no captan esta sección por su bajo potencial acuífero y amenaza de contaminación. Sin embargo, aquellos que lo captan, tratan de ubicar la rejilla en las secciones arenosas. El espesor de este lahar es variable, oscilando de 8 a 40 m (Is404). Posteriormente subyaciendo a este material aparece una lava de textura porfirítica, escoriácea, de permeablidad aparente muy alta y espesor de 6 a $10 \mathrm{~m}$. Esta lava es subyacida por una capa de arcilla de color rojo o café, que posiblemente constituye la sección superior de un lahar, el cual se identifica en las perforaciones Is- 226, Is-308, Is-304, Is-404 e Is-429. En este lahar se alternan tramos muy arcillosos con otros arenosos y ocasionales bloques ígneos, con una permeabilidad aparente de baja a muy baja. Las rejillas de los pozos permiten captar ciertos horizontes arenosos muy permeables. Las descripciones de los pozos Is-404 e Is-429, los más profundos, indican que aparecen lavas de color gris de dureza apreciable, fracturadas, infrayacidas por capas de toba, flujos de lavas, tobas y finalmente flujos de lava en las secciones más profundas de estas perforaciones.

Para el sitio Paraíso se han identificado 8 pozos que cuentan con información, Is-100, Is101, Is-103, Is-205, Is-390, Is-423, Is-435 e Is441 , los cuales suplen de agua subterránea a actividades agroindustriales y domésticas (Cuadro 4). El nivel estático reportado oscila entre 21,5 y $109 \mathrm{~m}$ de profundidad, ocurriendo este último en el pozo de mayor profundidad, el cual alcanza $148 \mathrm{~m}$. El caudal de extracción varía de 1,2 a 7,0 1/s, coincidiendo este último con el pozo de mayor profundidad, el cual posiblemente capta un nivel regional, mientras que los demás pozos captan acuíferos colgados. La transmisividad estimada en un pozo es de $120 \mathrm{~m}^{2} / \mathrm{d}$, y 
MORA \& VARGAS: Características geotécnicas e hidrogeológicas de sitios propuestos...

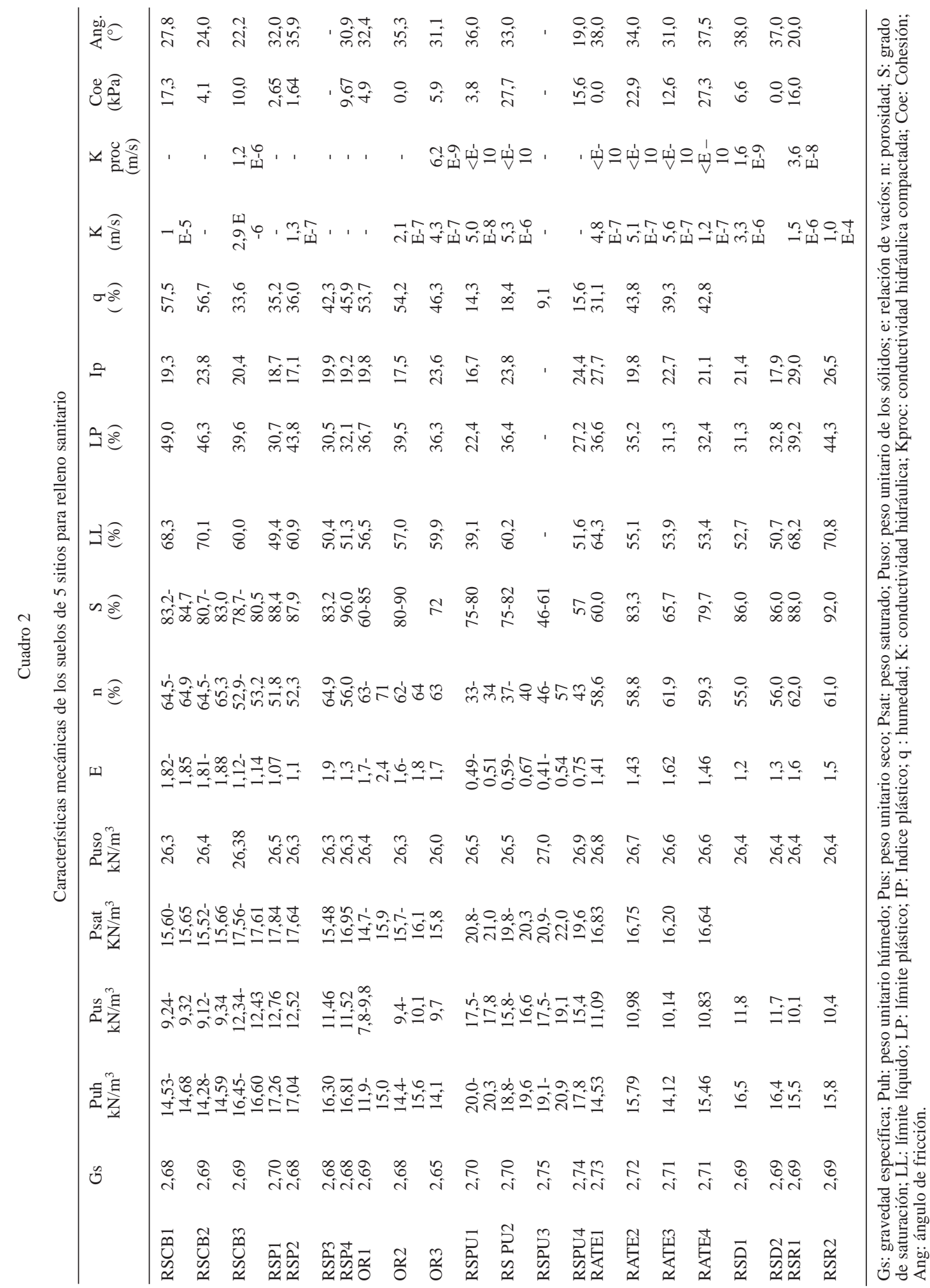


Cuadro 3

Características hidrogeológicas del sitio Oreamuno

\begin{tabular}{|c|c|c|c|c|c|c|c|c|c|}
\hline $\begin{array}{l}\text { Pozo } \\
(1 / \mathrm{s} / \mathrm{m})\end{array}$ & Coord. & Elev. & $\mathrm{NE}(\mathrm{m})$ & $\mathrm{Q}(1 / \mathrm{s})$ & Prof (m) & E.sat. (m) & $\mathrm{K}(\mathrm{m} / \mathrm{d})$ & $\mathrm{T}(\mathrm{m} 2 / \mathrm{d})$ & $\mathrm{Q} / \mathrm{s}(\mathrm{msnm})$ \\
\hline Is-226 & $\begin{array}{c}548,9- \\
205,3\end{array}$ & 1430 & 26 & 1,7 & 54,0 & 16,0 & 1,5 & 25 & 0,24 \\
\hline Is-304 & $\begin{array}{c}548,2- \\
206,6\end{array}$ & 1540 & 19,6 & 1,2 & 34,0 & 6,0 & 5,8 & $10-35$ & \\
\hline Is-308 & $\begin{array}{l}548,1- \\
206,9\end{array}$ & 1580 & 7,0 & 4,0 & 63,0 & 27,0 & 0,6 & 15 & 0,15 \\
\hline Is-309 & $\begin{array}{c}548,2- \\
206,8\end{array}$ & 1600 & 64,2 & 4,0 & 74,0 & & & 1244 & 9,4 \\
\hline Is-404 & $\begin{array}{c}549,1- \\
204,3\end{array}$ & 1400 & 147 & 14,2 & 200,5 & 38,0 & & & \\
\hline Is-429 & $\begin{array}{c}549,3- \\
205,4\end{array}$ & 1450 & 52,0 & 6,0 & 120,0 & 29,0 & & & \\
\hline
\end{tabular}

Cuadro 4

Características hidrogeológicas del sitio Paraíso

\begin{tabular}{|c|c|c|c|c|c|c|c|c|c|}
\hline Pozo & Coord. & $\begin{array}{c}\text { Elev. } \\
(\mathrm{msnm})\end{array}$ & $\mathrm{NE}(\mathrm{m})$ & $\mathrm{Q}(1 / \mathrm{s})$ & Prof (m) & E.sat. (m) & $\mathrm{K}(\mathrm{m} / \mathrm{d})$ & $\mathrm{T}(\mathrm{m} 2 / \mathrm{d})$ & $\mathrm{Q} / \mathrm{s}(1 / \mathrm{s} / \mathrm{m})$ \\
\hline Is- 100 & $\begin{array}{c}555,8- \\
202,8\end{array}$ & 1010 & & 1,13 & 27,0 & & & & \\
\hline Is-101 & $\begin{array}{l}556,1- \\
202,7\end{array}$ & 1010 & 21,5 & 1,3 & 30,0 & & & & \\
\hline Is-103 & $\begin{array}{l}555,6- \\
202,5\end{array}$ & 1000 & 25,0 & & 50,0 & & & & \\
\hline Is-205 & $\begin{array}{l}554,0- \\
203,2\end{array}$ & 1300 & & & 59,0 & & & & \\
\hline Is-390 & $\begin{array}{l}552,6- \\
203,8\end{array}$ & 1320 & 109,0 & 7,0 & 148,0 & & & & \\
\hline Is-423 & $\begin{array}{l}554,1- \\
205,7\end{array}$ & 1590 & & 5,0 & 50,0 & & & & \\
\hline Is-435 & $\begin{array}{c}555,9- \\
203,9\end{array}$ & 1060 & 29,0 & 1,9 & 69,0 & 28,0 & 14,2 & 120,0 & 0,97 \\
\hline Is-441 & $\begin{array}{c}203,5- \\
553,3\end{array}$ & 1330 & 38,0 & 3,2 & 66,0 & & & & \\
\hline
\end{tabular}

una capacidad específica de $0,97 \mathrm{l} / \mathrm{s} / \mathrm{m}$. Las perforaciones tienen profundidades que oscilan de 27 a $148 \mathrm{~m}$, siendo más frecuentes aquellas con profundidades menores de $70 \mathrm{~m}$. Utilizando la información que brindan algunas perforaciones, se extrae que una capa de suelo de $3 \mathrm{~m}$ aproximadamente cubre un depósito lahárico constituido por bloques de lava de tamaño variable, envuel- tos en una matriz arcillosa, de carácter plástico. Algunos pozos captan este material, que aunque presenta una matriz de baja permeabilidad, ocasionalmente aparecen lentes de arena, con permeabilidad aparente moderada, los cuales brindan escaso volumen de agua. El espesor aproximado de la capa es de 15 a $20 \mathrm{~m}$. Infrayaciendo este lahar aparece una lava, de color gris, densa, 


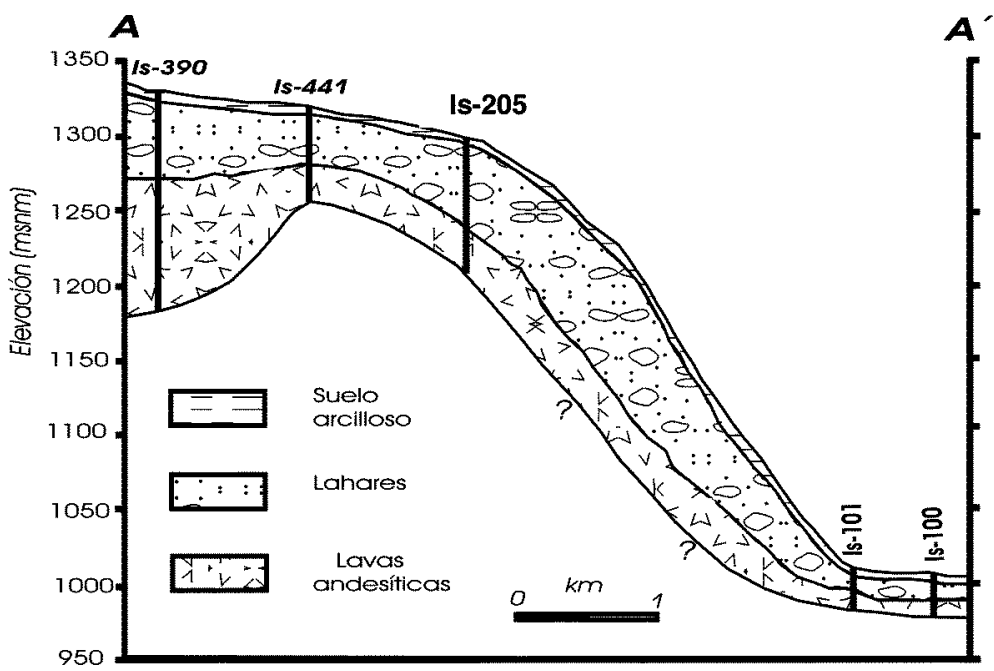

Fig. 2: Perfil esquemático de la estratigrafía en el área del relleno sanitario de Paraiso.

con algunos tramos fracturados de composición andesítica basáltica (Fig. 2). La permeabilidad aparente es alta. En la base de esta lava aparece una capa de toba arcillificada muy oxidada y de color rojo, de permeabilidad baja. Posteriormente aparece una lava de permeabilidad aparente de media a alta, de carácter andesítico y de textura vesicular, con un espesor de más de $10 \mathrm{~m}$. La mayoría de las perforaciones captan esta secciones lávicas, por medio de rejillas o tubería ranurada.

En las cercanías del sitio Santo Domingo, el cual es utilizado desde hace varios años por parte de la Municipalidad del mismo cantón para disponer desechos se encuentran los pozos AB-549, AB-656, entre otros, los cuales brindan información sobre las condiciones hidrogeológicas imperantes en el sitio. Es necesario destacar que varios pozos de abastecimiento, ubicados en el campo de pozos de la Valencia, se encuentran a una distancia de aproximadamente $1000 \mathrm{~m}$ desde el sitio de disposición de desechos y también se encuentran relativamente cerca los manantiales de La Libertad, que se utilizan para abastecer de agua potable un sector importante de la población.

Un estudio geofísico desarrollado por Leandro \& Arredondo (1996) para investigar las características geológicas e hidrogeológicas del cantón de Santo Domingo indican, a partir de sondeos eléctricos verticales (SEV), que la secuencia de rocas se inicia con suelos arcillosos con espesores de 25 a $30 \mathrm{~m}$, luego se encuentran tres flujos de lavas separados por tobas. Estos flujos de lavas podrían corresponder a las unidades hidrogeológicas La Libertad, Colima Superior y Colima inferior, de las más reciente a las más antigua (Fig. 3). Esta información se puede correlacionar con la información que brinda el pozo AB-549 (W15), en el cual se encuentran en orden descendente un paquete de tobas de $38 \mathrm{~m}$ de espesor, luego un acuífero colgado (La Libertad), tobas, un horizonte de lavas no saturada (Colima Superior) de $50 \mathrm{~m}$ de espesor, una toba de $9 \mathrm{~m}$ de espesor, un paquete de ignimbrita, confinada por tobas y finalmente el acuífero Colima Inferior con un espesor de $26 \mathrm{~m}$. Se encontraron 2 niveles de agua subterránea, uno de ellos ubicado a $48 \mathrm{~m}$ bajo el nivel de la superficie el cual corresponde a un nivel freático de Colima Superior y un nivel piezométrico a $80 \mathrm{~m}$ asociado al acuífero Colima Inferior. De acuerdo al Mapa Hidrogeológico del Valle Central Norte (SENARA-BGS, 1985), el nivel del agua subterránea del acuífero Colima Superior se encuentra aproximadamente a $90 \mathrm{~m}$ de profundidad en el sitio. El gradiente hidráulico en Colima Superior es de 


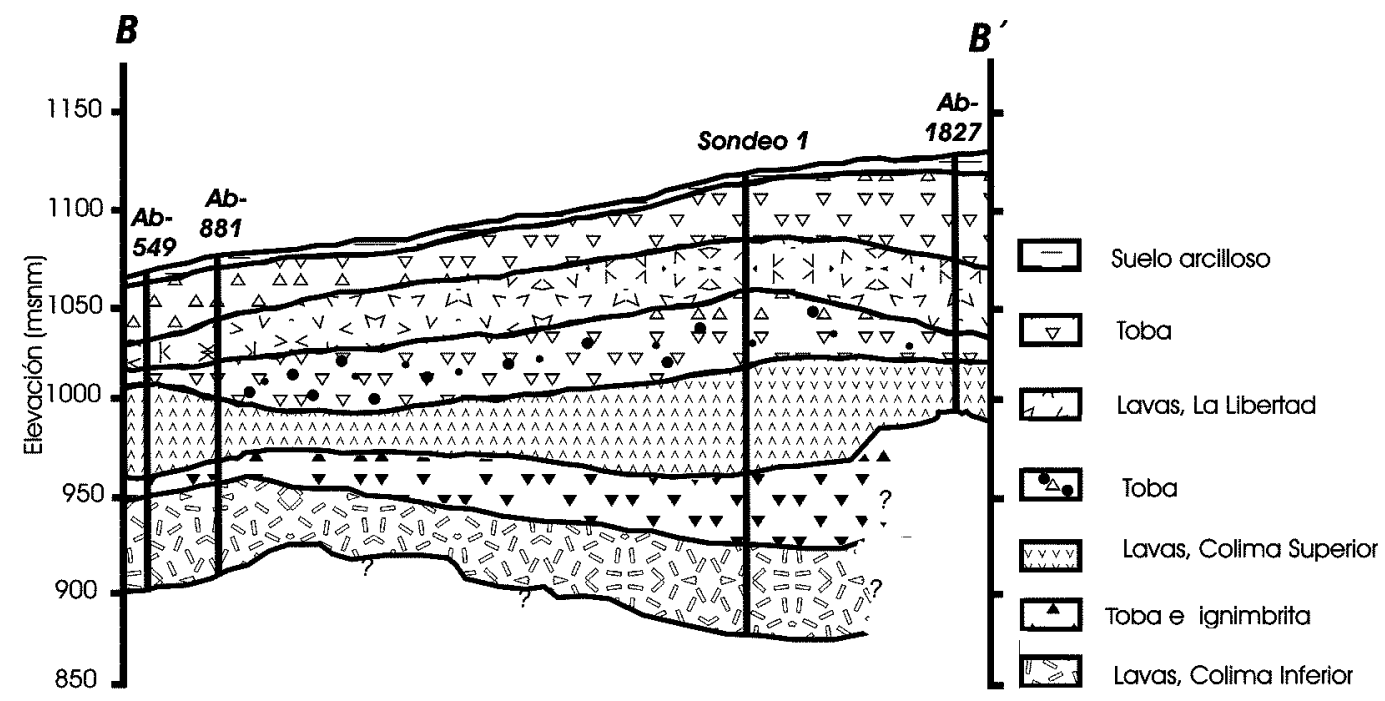

Fig. 3: Perfil esquemático de la estratigrafía en el área del relleno sanitario de Santo Domingo de Heredia.

aproximadamente $2 \%$, mientras que la conductividad hidráulica oscila de 500 a $9500 \mathrm{~m}^{2} / \mathrm{d}$ y el rendimiento específico es de 0,01 (Rodríguez, 1997).

Las unidades hidrogeológicas del sitio Pozos en Puriscal no se han logrado identificar por la inexistencia de perforaciones con información litológica. Por lo cual es necesario profundizar en las investigaciones sobre las condiciones hidrogeológicas locales, con la utilización de ensayos geofísicos o realizando perforaciones de pequeño diámetro.

Cerca del sitio Atenas se han identificado 9 perforaciones que abastecen algunas de las actividades que demandan agua en la ciudad de Atenas y también en las comunidades de Concepción y Pan de Azúcar (Cuadro 5). Los datos sobre estas perforaciones indican que el nivel estático se encuentra entre 6 y $35 \mathrm{~m}$ bajo la superficie del terreno.

Las perforaciones identificadas como RG370; RG-343; RG-10 y RG-132 se localizan a una distancia de $1000 \mathrm{~m}$ del sitio propuesto como relleno sanitario y cortan rocas de la Formación Grifo Alto, las que incluyen lavas andesíticas con cierto grado de meteorización. Las perforaciones identificadas como RG-461; RG-72; RG-453; RG-423 y RG-263, se encuentran al sur, en las rocas de la Formación Avalancha Ardiente, las que incluye tobas e ignimbritas. Por medio de la ubicación de los pozos y de la interpretación de la elevación del nivel de agua subterránea, se considera la existencia de dos acuíferos (Mora et al., 2000). Uno de ellos se localiza al Norte del posible relleno y está captado por los pozos RG370; RG-343; RG-10 y RG-132, mientras que el otro se localiza al sureste del sitio y es captado por los pozos RG-461; RG-72, RG-453, RG-423 y RG-263. Se asume que las líneas de flujo en el acuífero del norte tienen una dirección precisamente hacia esa misma dirección.

En las inmediaciones del sitio propuesto para relleno sanitario en Bolívar de San Ramón, se han identificado cuatro pozos de abastecimiento de agua, denominados Na-167, Na-511, Na-547 y Na-554, ubicados estos dos últimos pozos en la localidad de Bolivar. El nivel estático medido en los pozos varía de 51 a $62 \mathrm{~m}$, mientras que la transmisividad y la capacidad específica varían de 12 a $25 \mathrm{~m}^{2} / \mathrm{d}$ y 0,16 a $0,36 \mathrm{l} / \mathrm{s} / \mathrm{m}$ respectivamente (Cuadro 6).

La profundidad máxima alcanzada es de 114 m en el pozo Na-554; sin embargo, su caudal es de solo 1,8 1/s, inferior al caudal medio en los otros pozos. Por su parte en los pozos Na-511 y 
Cuadro 5

Características hidrogeológicas del sitio Atenas

\begin{tabular}{|c|c|c|c|c|}
\hline Pozo & Coordenadas & Elev. (msnm) & $\mathrm{NE}(\mathrm{m})$ & Prof (m) \\
\hline RG-370 & $494,70-217,50$ & 700 & 15 & 50 \\
\hline RG-343 & $495,45-217,45$ & 680 & 8 & 45 \\
\hline RG-10 & $494,50-217,22$ & 710 & 10 & 65 \\
\hline RG-132 & $494,90-217,70$ & 680 & 23 & 43 \\
\hline RG-461 & $496,60-216,00$ & 535 & 23 & 60 \\
\hline RG-72 & $496,60-216,0$ & 535 & 35 & 52 \\
\hline RG-453 & $496,70-216,50$ & 560 & 6 & 26 \\
\hline RG-423 & $495,30-214,70$ & 520 & 15 & 42 \\
\hline RG-263 & $495,65-214,30$ & 470 & 8 & 30 \\
\hline
\end{tabular}

Cuadro 6

Características hidrogeológicas del sitio Bolivar, San Ramón

\begin{tabular}{|c|c|c|c|c|c|c|c|c|c|}
\hline Pozo & Coord. & $\begin{array}{c}\text { Elev. } \\
(\mathrm{msnm})\end{array}$ & $\mathrm{NE}(\mathrm{m})$ & $\mathrm{Q}(1 / \mathrm{s})$ & Prof (m) & E.sat. (m) & $\mathrm{K}(\mathrm{m} / \mathrm{d})$ & $\mathrm{T}(\mathrm{m} 2 / \mathrm{d})$ & $\mathrm{Q} / \mathrm{s}(1 / \mathrm{s} / \mathrm{m})$ \\
\hline $\mathrm{Na}-511$ & $\begin{array}{c}482,4- \\
232,5\end{array}$ & 1110 & 51,0 & 3,0 & 95,0 & 39,0 & 0 & 19 & 0,6 \\
\hline $\mathrm{Na}-547$ & $\begin{array}{l}481,8- \\
231,6\end{array}$ & 1960 & 60,0 & 2,5 & 75,0 & 15,0 & 0,8 & 12 & 0,21 \\
\hline $\mathrm{Na}-554$ & $\begin{array}{c}482,7- \\
232,8\end{array}$ & 1100 & 62,0 & 1,8 & 114,0 & 52,0 & 0,5 & 25 & 0,36 \\
\hline Na-167 & $\begin{array}{c}483,8- \\
231,7\end{array}$ & 1210 & & 1,1 & 81,0 & & & & \\
\hline
\end{tabular}

Na 547 se reporta una capa de arcilla, de color café, plástica y relativamente impermeable, cuyo espesor varía de 6 a $10 \mathrm{~m}$. Posteriormente aparece una toba formada por ceniza, la cual se asocia con una lava meteorizada (Na-547). Este material tiene un espesor aproximado de $40 \mathrm{~m}$ y es subyacido por una lava con una permeabilidad aparente de media a alta, la cual permite la circulación de agua, ya que se reportan planos de oxidación; se estima que cuenta un espesor aproximado de $40 \mathrm{~m}$.

Aunque la perforación Na-554 está relativamente cerca de la $\mathrm{Na}-511$ se reporta que solamente se encontró una unidad litológica, constituida por una secuencia de tobas de varios colores; sin embargo, a partir de los $60 \mathrm{~m}$ de profundidad el material se vuelve más oscuro, sano y de mayor dureza y corresponde con la zona productora de agua del pozo. Esta última característica coincide con la unidad de lavas reportada en los otros dos pozos a una profundidad similar. Las unidades hidrogeológicas captadas corresponden, en primer lugar, a las lavas, sin embargo las tobas meteorizadas son captadas en algunos tramos de la perforación, ya que tienen una conductividad hidráulica aparente media.

Para el sitio cerro Botadero en las Juntas de Abangares se han identificado 6 pozos que cuentan con información: Ju-5, Ju-11, Ju-12, Ju-13, Ju16, Ju-19. El nivel estático reportado oscila entre 4,3 y $8 \mathrm{~m}$ de profundidad, presentándose de manera relativamente superficial, lo cual incide sobre el potencial de contaminación. El caudal de extracción no supera los 2,5 1/s. La transmisividad 
Cuadro 7

Características hidrogeológicas del sitio Cerro Botadero

\begin{tabular}{|c|c|c|c|c|c|c|c|c|c|}
\hline Pozo & Coord. & $\begin{array}{c}\text { Elev. } \\
(\mathrm{msnm})\end{array}$ & $\mathrm{NE}(\mathrm{m})$ & $\mathrm{Q}(1 / \mathrm{s})$ & Prof (m) & E.sat. (m) & $\mathrm{K}(\mathrm{m} / \mathrm{d})$ & $\mathrm{T}(\mathrm{m} 2 / \mathrm{d})$ & $\mathrm{Q} / \mathrm{s}(1 / \mathrm{s} / \mathrm{m})$ \\
\hline Ju-5 & $\begin{array}{c}430,1- \\
251,0\end{array}$ & 150 & 6,0 & 2,5 & 35,0 & 5 & 40 & 200 & \\
\hline Ju-11 & $\begin{array}{c}429,9- \\
250,9\end{array}$ & 110 & & & & & & & \\
\hline $\mathrm{Ju}-12$ & $\begin{array}{c}429,0- \\
249,9\end{array}$ & 140 & 8,0 & 1,0 & 26,0 & 25 & 0,7 & 20 & 0,14 \\
\hline $\mathrm{Ju}-13$ & $\begin{array}{c}430,8- \\
250,1\end{array}$ & 140 & & & & & & & \\
\hline Ju-16 & $\begin{array}{c}432,4- \\
249,9\end{array}$ & 170 & 4,3 & & 30,0 & & & & \\
\hline Ju-19 & $\begin{array}{l}429,8- \\
250,2\end{array}$ & 150 & & & 26,0 & & & & \\
\hline
\end{tabular}

estimada varía de 20 a $200 \mathrm{~m}^{2} / \mathrm{d}$, y una capacidad específica de $0,14 \mathrm{l} / \mathrm{s} / \mathrm{m}$. Las perforaciones tienen profundidades que oscilan de 26 a $38 \mathrm{~m}$ (Cuadro 7). Los pozos Ju-12, Ju-13, Ju-16 y Ju-19 se localizan en el valle coluvio-aluvional del río San Juan, donde también se encuentran los cauces intermitentes de las quebradas Gata y Seca. El pozo 5 se ubica en el valle del río Abangares. Los dos valles se encuentran separados por una serranía con orientación E-W, la cual cuenta con una elevación máxima de 345 msnm en el cerro Botadero.

Analizando los registros de pozos, se puede concluir que en el valle del río San Juan existe un acuífero libre, el cual está siendo captado por el pozo Ju-16 y un acuífero confinado captado por el pozo Ju-12. Por su parte en el valle del río Abangares se cuenta con un acuífero libre, compuesto por materiales coluvio-aluvionales.

\section{ALGUNAS CONSIDERACIONES REGLAMENTARIAS SOBRE LOS RELLENOS SANITARIOS}

El reglamento sobre rellenos sanitarios del 22 de octubre de 1993 vigente en nuestro país, establece dos tipos de rellenos sanitarios: el manual y el mecanizado. El relleno manual se utilizará como método de disposición final de los desechos ordinarios de poblaciones urbanas y rurales que generan menos de 20 toneladas diarias. El relleno mecanizado se utiliza como método de disposición final para aquellas poblaciones que generen más de 40 toneladas diarias. Para las poblaciones que generen entre 20 y 40 pueden utilizar cualquiera de las dos. Además señala que los taludes finales no deben tener una inclinación mayor a $30 \%$. La base de suelo impermeable debe tener una permeabilidad no superior a los $10^{-8} \mathrm{~m} / \mathrm{s}$, un espesor mínimo de $50 \mathrm{~cm}$ y compactación al $95 \%$ del proctor estándar y con pendiente mínima de la base del 3\% hacia las líneas de los tubos de drenaje. El control de la calidad del agua subterránea se debe realizar mediante la perforación de al menos 3 pozos para así detectar la posible presencia de contaminación por la operación del relleno. La disposición de los desechos se debe ejecutar en capas de $60 \mathrm{~cm}$ de espesor y compactar cada capa mediante un mínimo de cuatro pasadas con maquinaria de un peso mínimo de 15 toneladas.

\section{EVALUACIÓN DEL POTENCIAL DE CONTAMINACIÓN DEL AGUA SUBTERRÁNEA}

La vulnerabilidad a la contaminación de los acuíferos puede ser evaluada usando la metodología propuesta por Le Grand (en Todd, 1976), en la cual se consideran la distancia desde el 
Cuadro 8

Potencial de contaminación de los sitios propuestos como rellenos sanitarios

\begin{tabular}{|c|c|c|c|c|c|c|c|}
\hline Sitio & $\begin{array}{l}\text { Prof. } \\
(\mathrm{m})\end{array}$ & Absorción & $\mathrm{K}(\mathrm{m} / \mathrm{d})$ & $\begin{array}{c}\text { Gradiente } \\
(\%)\end{array}$ & $\begin{array}{l}\text { Dis.Horiz. } \\
\quad(\mathrm{m})\end{array}$ & Total & $\begin{array}{c}\text { Pot. } \\
\text { Contam. }\end{array}$ \\
\hline Paraíso & $\begin{array}{l}21,5 \\
(6,5)\end{array}$ & $\begin{array}{c}\text { Arcilla y arena } \\
(5,0)\end{array}$ & Gravas $(0,5)$ & $4(2)$ & $500(9,5)$ & 23,5 & $\begin{array}{c}\text { Muy } \\
\text { improbable }\end{array}$ \\
\hline Oreamuno & $7,0(3,0)$ & $\begin{array}{c}\text { Arcilla y arena } \\
(5,0)\end{array}$ & $\begin{array}{c}\text { Arena y arcilla } \\
(0,5)\end{array}$ & $8(1,5)$ & $1500(10,5)$ & 21 & $\begin{array}{c}\text { Muy } \\
\text { improbable }\end{array}$ \\
\hline Santo Domingo & $48(8,5)$ & $\begin{array}{c}\text { Arcilla y arena } \\
(2,5)\end{array}$ & Grava (0) & $2(2,5)$ & $1000(10)$ & 23,5 & $\begin{array}{c}\text { Muy } \\
\text { improbable }\end{array}$ \\
\hline Atenas & $6(2)$ & $\begin{array}{c}\text { Arena y arcilla } \\
(2,5)\end{array}$ & $\begin{array}{c}\text { Toba y arena } \\
(2,5)\end{array}$ & $3,5(2,5)$ & $1000(10)$ & 19,5 & $\begin{array}{c}\text { Muy } \\
\text { improbable }\end{array}$ \\
\hline Bolivar & $51(8,5)$ & $\begin{array}{l}\text { Arcilla } \\
(5,5)\end{array}$ & $\begin{array}{c}\text { Toba y arena } \\
(2,5)\end{array}$ & $2(2,5)$ & $500(9,5)$ & 30 & $\begin{array}{c}\text { Muy } \\
\text { improbable }\end{array}$ \\
\hline Cerro Botadero 4 & $4,3(1,5)$ & Arena (2) & $\begin{array}{c}\text { Arenas y gravas } \\
(0,5)\end{array}$ & $5(2,0)$ & $1570(10,5)$ & 16,5 & $\begin{array}{c}\text { Muy } \\
\text { improbable }\end{array}$ \\
\hline
\end{tabular}

relleno sanitario hasta el nivel freático, el gradiente hidráulico, la cercanía a pozos de abastecimiento de agua y las características de la zona no saturada para atenuar los contaminantes.

La profundidad al nivel freático es un factor importante, ya que a una profundidad escasa se presentarán tiempos más cortos de recorrido de los contaminantes, generándose solo una pequeña atenuación antes de que los contaminantes alcancen el acuífero. Con respecto al gradiente hidráulico, en el sitio es recomendable que el nivel freático se incline en contra de las fuentes locales de abastecimiento. Cuanto menos inclinado sea el gradiente, el tiempo de atenuación es mayor. El acuífero debe ser considerado como un recurso muy valioso, que debe ser protegido de la contaminación para el uso presente y futuro, sobre todo cuando es de buena calidad.

Los resultados obtenidos se han obtenido luego de integrar los valores de cada parámetro, señalados por la metodología de Le Grand, el cual sin embargo no considera las condiciones de recarga y de pluviometría, que pueden facilitar la generación de lixiviados. De hecho, las precipitaciones en el sector de San Ramón y de Cartago son más elevadas que en sector de las Juntas de Abangares, lo que influiría sobre el potencial de contaminación. Además, el tipo de desechos y la producción de los mismos no son similares, ya que en los sectores rurales la cantidad de dese- chos orgánicos es mayor, mientras que el volumen total producido es menor que en otras regiones urbanas o semiurbanas. Por último, es necesario señalar que en el cuadro 8 entre paréntesis se muestran los valores asignados de acuerdo a la puntuación otorgada por la metodología aplicada.

Además, en el caso de los valores de conductividad hidráulica (k) del acuífero, su valor se ha estimado de la carta de evaluación de potencial, asimilando las características hidrogeológicas obtenidas de los datos de campo y de las perforaciones con los materiales utilizados en dicha carta (Fig. 4).

\section{CONSIDERACIONES FINALES}

Un sistema adecuado de impermeabilización de la base constituye una protección contra la contaminación del agua subterránea, aunque es necesario investigar con más profundidad las características de retardación de diferentes contaminantes en los diferentes suelos utilizados. Además, se debe analizar la influencia de la compactación del suelo en el proceso de retardación.

En el caso del sitio Paraíso es difícil establecer un criterio uniforme para realizar la compactación, ya que no se conoce la distribución completa de los materiales, por lo cual se 

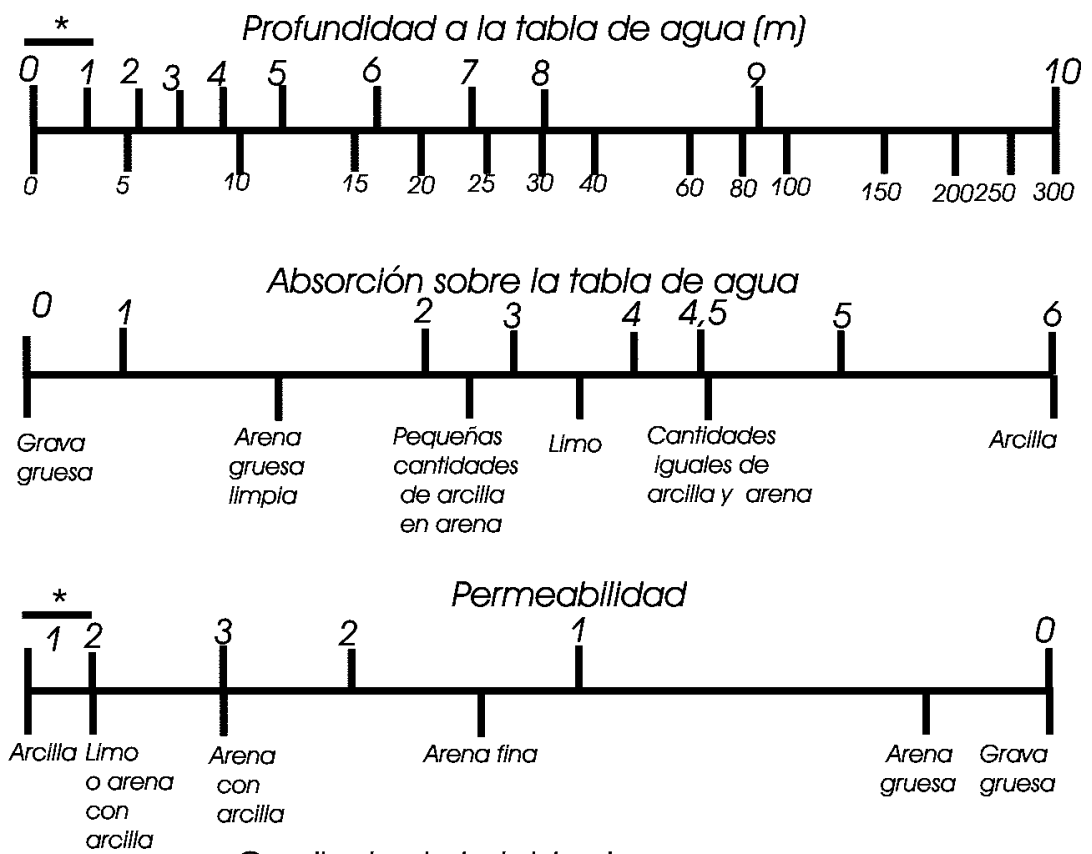

Gradiente de la tabla de agua
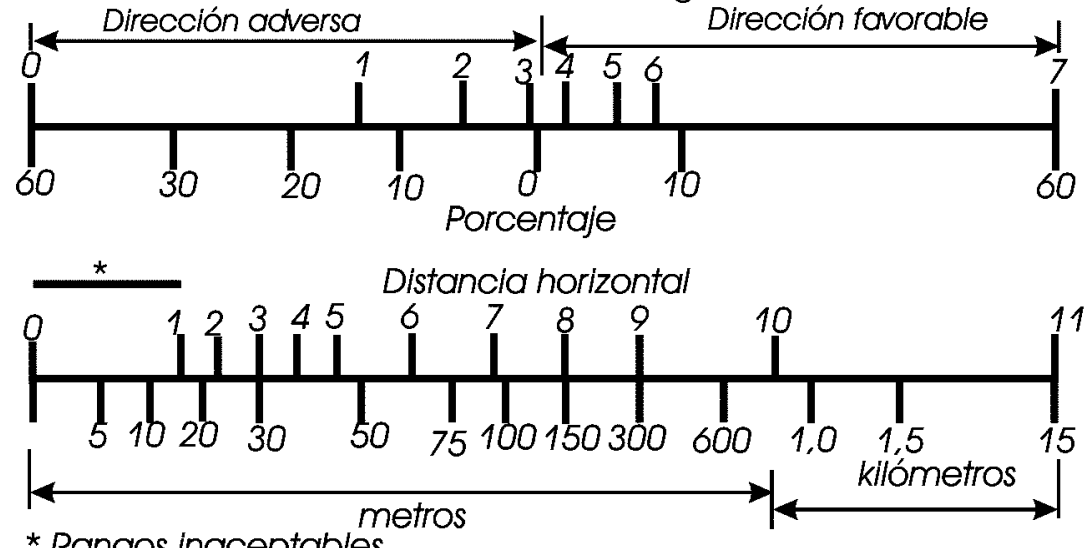

Fig. 4: Carta de evaluación del potencial de contaminación de Le Grand.

recomienda realizar más ensayos de compactación con el material que se desea utilizar.

Las características de compactación de los tres materiales encontrados durante las excavaciones en el sitio Oreamuno, son relativamente similares. Por lo que podrían utlizarse incluso mezclados, siempre y cuando el contenido de humedad se mantenga en el rango de 37,5 a $44,5 \%$. Durante la operación del relleno, las paredes ver- ticales de las trincheras no deberían tener más de 2,7 m de altitud; sin embargo, en términos generales se recomienda la utilización de un talud de $60^{\circ}$ de inclinación, con altura máxima de $5,5 \mathrm{~m}$.

El sitio Santo Domingo, reviste gran importancia, ya que se ubica cerca del campo de pozos de la Valencia y de las fuentes de la Libertad, que abastecen gran parte de la población de la gran área metropolitana. Sin embargo, al ser un 
basurero que se encuentra en funcionamiento desde hace varios años, el diseño de la impermeabilización de la base y las medidas de protección deben enfocarse de manera diferente que los otros sitios analizados. Como una primera medida se deberían establecer piezómetros de monitoreo gradiente abajo del sitio para evaluar periódicamente la calidad del agua subterránea, además, colocar capas de arcilla compactada separando las celdas de desechos.

Los suelos residuales de la Formación Peña Negra, en el sitio Pozos de Puriscal, pueden utilizarse para impermeabilizar ya que su resistencia al corte en estado compactado es media y su resistencia a la compresibilidad es alta. $\mathrm{La}$ permeabilidad de los materiales varía de $5 \times 10^{-4} \mathrm{a}$ $5 \times 10^{-6} \mathrm{~cm} / \mathrm{s}$, lo que significa que son poco permeables, sin embargo se debe considerar que estos materiales se encuentran sobre rocas intensamente fracturadas y de permeabilidad más alta. Por lo tanto, para efectos de diseño se deben tomar en cuenta varias alternativas para impermeabilizar los sitios donde se coloquen los desechos para evitar la contaminación. La compactación adecuada de los materiales se puede lograr, si la humedad se mantiene entre el 19 y $27 \%$. Con los parámetros de resistencia al corte y considerando que el espesor de suelo máximo observado fue de 2,3 m, se pueden construir taludes con un ángulo de inclinación de alrededor de $70^{\circ}$ para la primera secuencia de materiales. Alrededor de este sitio se han identificado varias fuentes sísmicas de gran potencial, por lo que se deben tomar las medidas para minimizar el riesgo de apertura de fracturas y la fuga de lixiviados.

En el caso del sitio Atenas deben manejarse con sumo cuidado la disposición de desechos y la impermeabilización adecuada para evitar la contaminación directa de las quebradas que nacen en la fila Boquerón y que presentan un patrón de drenaje radial y cuyo origen está muy cerca del sitio propuesto como relleno sanitario.

El sitio denominado cerro Botadero en las Juntas de Abangares, presenta un potencial de contaminación alto, ya que a cierta distancia se encuentra un acuífero superior muy permeable, el nivel de agua se encuentra relativamente cerca de la superficie y la ubicación del sitio es desfavorable. Por lo tanto se recomienda realizar una impermeabilización adecuada para disminuir el potencial de contaminación.

En varios de los sitios estudiados la conductividad hidráulica de los materiales muestreados se reduce en dos órdenes de magnitud durante el proceso de compactación lo que permite utilizarlos como material de impermeabilización de la base. Solamente una muestra del cerro Botadero no ha alcanzado el valor requerido después de la campactación.

Aunque el potencial de contaminación estimado para los sitios analizados es bajo, es necesario llevar a cabo impermeabilizaciones de la base y del techo de las celdas de desechos que minimizen el paso de los contaminantes hacia el agua subterránea. Además se deben realizar ensayos de secado y de humedecimiento, para medir el nivel de retracción de las arcillas sobre todo en regiones con temperaturas del aire elevadas durante la época seca, como es el caso del sitio cerro Botadero, ya que estas grietas podrían ser una vía de transporte de los lixiviados. Además se debe tener en cuenta que la carta de evaluación de contaminación de Le Grand está diseñada para acuíferos libres en materiales aluvionales lo cual sobreestima los valores asignados a los diferentes parámetros.

Los sitios elegidos no siempre cumplen con los requisitos adecuados para evitar la contaminación del agua subterránea, sin embargo existen limitaciones financieras que impiden a los municipios adquirir terrenos con características idóneas, y se deberían entonces implementar impermeabilizaciones de la base con controles geotécnicos adecuados, que se llevarían a cabo por medio de la sección de hidrogeología y geotecnia de la Escuela de Geología o de otros entes especializados. No obstante los ayuntamientos deberían implementar programas de reutilización y reciclaje así como de compostaje de los desechos.

\section{AGRADECIMIENTOS}

Agradecemos a los evaluadores que externaron sus críticas y sugerencias. Este trabajo se ha desarrollado bajo el proyecto de investigación "Manejo Aadecuado de los recursos hídricos subterráneos en Costa Rica“, inscrito ante la Vicerrectoría de Investigación de la Universidad de Costa Rica. 


\section{REFERENCIAS}

ALVARADO, E., 1994: Estudio geológico, ambiental y estratigrafía de la región oeste de San Ramón. - 55 págs. Univ. de Costa Rica, San José [Inf. Campaña Geol.].

ARIAS, O, 1988: Geología de pozos de Puriscal y alrededores, San José. - 14 págs. Univ. de Costa Rica, San José [Inf. Campaña Geol.].

DENYER, P \& ARIAS, O., 1990: Geología de la hoja Candelaria. - Escala 1: 50 000, IGN, San José.

DENYER, P \& ARIAS, O., 1991: Estratigrafía de la región central de Costa Rica. - Rev. Geol. América Central, 12: $1-59$.

ECHANDI, E., 1981: Unidades volcánicas de la vertiente norte de la cuenca del río Virilla. - 123 págs. Univ. de Costa Rica, San José [Tesis Lic.]."

KNOBLICH, K., \& MANDS, E., 1993: Discusión sobre los sitios para los vertederos de desechos sólidos de San José. - Rev. Geol. América Central, 16: 95-101.

KRUSHENSKY, R.D., 1972: Geology of the Istarú Quadrangle, Costa Rica. - 46 págs. Geol. Surv. Bull. 1358.

LA NACIÓN, 12 de agosto de 2000: Rellenos sanitarios. Pág. 6A El País.

LA NACIÓN, 15 de agosto de 2000: Garabito con relleno. Pág. 8A El País.

LEANDRO, G., \& REDONDO, C., 1996: Estudio geofísico mediante geoeléctrica en el cantón de Santo Domingo y alrededores. - [sin \# págs.] Escuela de Geología, Univ. de Costa Rica, San José [Informe interno].
MADRIGAL, O., 1980: Geología y potencial de una parte del distrito minero de Abangares. - 84 págs. Univ. de Costa Rica, San José [Tesis Lic.].

MONTERO, W., 1994: Sismicidad y neotectónica. - En: DENYER P. \& KUSSMAUL, S. (eds.): Atlas geológico de la gran Área Metropolitana: 147-160. Ed. Tecnológica, Cartago.

MORA, R., 1998: Propiedades físicas, hidráulicas y mecánicas de suelos de origen volcánico, en sitios seleccionados del Valle Central, Costa Rica. - Rev. Geol. América Central, 19: 81-98.

MORA, R., CASTRO, A., RAMOS, V. \& BADILLA, E., 2000: Características geotécnicas del relleno sanitario de Atenas. - 20 págs. Esc. de Geología, Univ. de Costa Rica, San José [Informe interno].

MORALES, L. \& AGUILAR, A., 1994: Amenaza sísmica. En: DENYER, P. \& KUSSMAUL, S. (eds.): Atlas geológico de la gran Área Metropolitana: 233-243, Ed. Tecnológica, Cartago.

RODRÍGUEZ, H., 1997: Acuíferos Volcánicos de Costa Rica. - En: MORA, R. (ed.): Los acuíferos volcánicos y el desarrollo sostenible en Centroamérica. - 52 págs. Escuela de Geología, Univ. de Costa Rica, San José [Informe interno].

SENARA \& BGS, 1985: Mapa hidrogeológico del Valle Central de Costa Rica. - Escala 1:50 000, SENARABGS, Gran Bretaña.

TODD, D.K., 1976 : Groundwater hydrology. - [2 ed.] 535 págs. Wiley. New York.

WALTON; W., 1970: Groundwater resource evaluation. - 664 págs. Mc Graw-Hill, New York. 